\section{Bandpass filter implemented with blazed waveguide sidewall gratings in silicon-on-insulator}

A.V. Velasco, P.J. Bock, P. Cheben, M.L. Calvo, J.H. Schmid, J. Lapointe, D.-X. Xu, S. Janz and A. Delâge

The fabrication and experimental characterisation of a two-stage bandpass filter based on curved waveguide sidewall gratings is reported for the silicon-on-insulator platform. At each cascaded filtering stage, the spectral components of the input signal are dispersed by the diffraction grating formed in the sidewall of a silicon strip waveguide. Different wavelengths are focused onto different positions along the Rowland circle and the filter central wavelength is selected by a specific receiver waveguide. By using two consecutive filtering stages, both the filter passband profile and the stopband rejection ratio are substantially increased. The grating is apodised and chirped to ensure a constant effective index along the grating length to minimise phase distortions. Blazed geometry is used to maximise the diffraction efficiency to the -1 st order. The device was fabricated with electron beam lithography and reactive ion etching using a single etch step. A bandwidth of $6.2 \mathrm{~nm}$ was measured near $1590 \mathrm{~nm}$ for the fabricated filter, with a roll-off of $4 \mathrm{~dB} / \mathrm{nm}$ at the passband edge, and a stopband rejection of $40 \mathrm{~dB}$.

Introduction: Silicon-on-insulator (SOI) high-index contrast waveguide technology enables the development of compact photonic devices with large integration densities, for applications including optical interconnects, telecommunications, biological sensing, nonlinear photonics and spectroscopy. These applications often require highly selective wavelength filtering with a small device footprint. Filters implemented with Bragg gratings [1] have been proposed, although multimode interference (MMI) couplers are required to extract the reflected signal. Ring resonators [2] exhibit a great wavelength selectivity, but their resonance conditions are highly dependent of fabrication errors and temperature variations. Other devices with larger footprints have also been implemented, such as arrayed waveguide gratings (AWGs) and echelle gratings [3], or Mach-Zehnder interferometer (MZI) lattice filters [4]. However, it remains a challenge to develop compact bandpass filters which operate in a transmission configuration and present a high stopband rejection.

The use of curved waveguides with UV induced gratings to achieve wavelength dispersion was theoretically proposed [5] to develop wavelength division multiplexing (WDM) systems more compact than AWGs and echelle gratings [3]. A new type of planar waveguide multiplexer has been proposed, based on a curved SOI sidewall waveguide grating with a blazed grating profile [6], having a simulated crosstalk of $-30 \mathrm{~dB}$, an operational range exceeding $370 \mathrm{~nm}$ and a layout size of only $90 \times 140 \mu \mathrm{m}$. In this Letter, we adapt this sidewall waveguide grating wavelength multiplexer design [6] to achieve a highly selective bandpass filter with two cascaded stages.

Principle of operation and device design: At each stage of the bandpass filter, light is coupled into a curved silicon strip waveguide and diffracted by a sidewall grating. The diffracted light is coupled into a slab waveguide where different wavelengths are focused on different positions along the focal curve defined by the Rowland circle geometry. Light at the central wavelength $\lambda_{0}=\Lambda / n_{\text {eff }}$ is collected by the receiver waveguide, where $\Lambda$ is the grating pitch and $n_{\text {eff }}$ is the effective index of the fundamental mode of the input waveguide. By judiciously designing the grating pitch and the position of the receiver waveguide, only a narrow wavelength band is transmitted. By cascading two filtering stages of the same characteristics, the resulting passband is narrowed and the stopband suppression is increased. The receiver waveguide of the first stage is coupled directly to the second stage waveguide sidewall grating, preserving a small footprint. The waveguide grating teeth are blazed in order to maximise the optical power diffracted towards the receiver waveguides of the -1 st diffracted order.

At both filtering stages the blazed sidewall grating has a length of $100 \mu \mathrm{m}$ and a width of $0.6 \mu \mathrm{m}$. The blazed diffraction grating is etched in the outer sidewall of the curved input waveguide, with a maximum modulation depth of $0.3 \mu \mathrm{m}$. Previous finite difference time domain (FDTD) simulations determined that over $99 \%$ of the optical power is diffracted from the input waveguide along the sidewall grating length of $100 \mu \mathrm{m}$. The diffracted light is coupled into the slab waveguide through a $1 \mu \mathrm{m}$-wide subwavelength antireflective (AR) grating boundary [7], which comprises a triangular-shaped impedance matching structure with a pitch of $0.3 \mu \mathrm{m}$. The receiver waveguide is $1.4 \mu \mathrm{m}$ wide and is placed at a distance of $70 \mu \mathrm{m}$ from the diffraction grating at the centre of the arc formed by the curved waveguide. These parameters ensure the matching of the numerical apertures of the receiver waveguide and the diffraction grating, as previously computed with FDTD simulations.

To suppress secondary diffraction peaks, and to obtain a near-field Gaussian profile, apodisation was applied over the first $70 \mu \mathrm{m}$ of the diffraction grating following a Gaussian apodisation function $y=$ $y_{0} \exp \left(-x^{2} / 2 \sigma^{2}\right)$, where $y_{0}$ is the maximum modulation depth of $0.3 \mu \mathrm{m}$. A value of $\sigma=60 \mu \mathrm{m}$ was determined from simulations. Phase errors were corrected by chirping the grating pitch along the waveguide, thus ensuring a constant effective index and phasefront tilt along the grating length. FDTD simulations were performed to determine the grating pitch required to obtain a far-field peak angle of $0^{\circ}$ for each modulation depth. This dependency was fitted to a polynomial function, which was applied to the final grating design, resulting in a grating pitch ranging from 560 to $590 \mathrm{~nm}$. Both the intensity and the phase distributions of the near-field profile can be controlled by using apodisation and chirping of the waveguide sidewall grating, thus allowing tailoring the shape of the filter passband. This is an important design advantage compared to AWGs and echelle gratings where altering the near-field intensity profile implies incurring a loss penalty [3].

Fabrication and characterisation: Samples were fabricated on SOI substrates with a $0.26 \mu \mathrm{m}$-thick silicon and $2 \mu \mathrm{m}$-thick buried oxide layer. A single patterning step by electron beam lithography with high contrast hydrogen silsesquioxane (HSQ) resist was used to define the structure of the device. The resist pattern was transferred into the silicon layer by inductively coupled plasma reactive ion etching (ICP-RIE). An optical micrograph image of the fabricated filter is shown in Fig. 1.

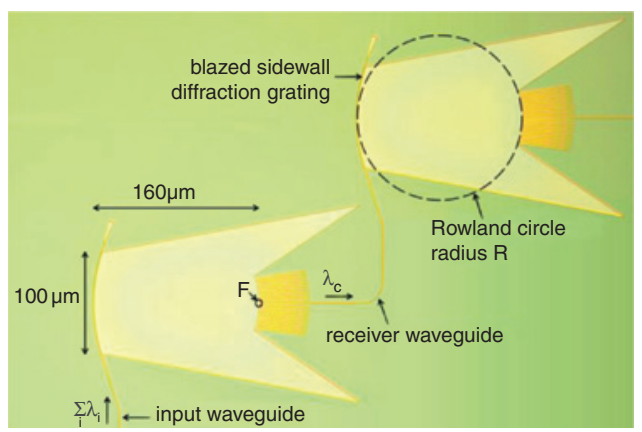

Fig. 1 Optical micrograph image of fabricated two-stage filter

Central wavelength $\lambda_{c}$ focused in position $\mathrm{F}$ of Rowland circle and guided to the input of the second filtering stage

The spectral response of bandpass filters with designed central wavelengths ranging from 1520 to $1590 \mathrm{~nm}$ were characterised using a tunable laser with a wavelength range of 1500-1620 nm. Polarisation control optics were used to ensure transverse electric (TE) input polarisation. The device temperature was thermoelectrically stabilised using a Peltier module. A subwavelength grating mode transformer was included at the chip input and output facets for efficient light coupling with a lensed optical fibre.

Results: The normalised transmission spectrum of the fabricated device is shown in Fig. 2, for a device temperature of $23.5^{\circ} \mathrm{C}$. A near-Gaussian profile with no sidelobes is observed. A central wavelength of $1589.6 \mathrm{~nm}$ and $-3 \mathrm{~dB}$ bandwidth of $6.2 \mathrm{~nm}$ are obtained. Both sides of the passband are followed by a steep slope with a $4 \mathrm{~dB} / \mathrm{nm}$ roll-off that reaches a white background noise region with a stopband rejection ranging from -35 to $-45 \mathrm{~dB}$ for wavelengths separated $\geq 12.5 \mathrm{~nm}$ from the central wavelength. The shape factor, defined as the ratio of the $-40 \mathrm{~dB}$ bandwidth to the $-3 \mathrm{~dB}$ bandwidth is 4.03 , showing a high skirt selectivity. A $12 \mathrm{~dB}$ improvement in the stopband rejection is achieved compared with a single-stage filter of similar design, as well as a $30 \%$ reduction in the $-3 \mathrm{~dB}$ bandwidth. Intrinsic losses of $3.5 \mathrm{~dB}$ were measured for each filtering stage at the central wavelength. The temperature dependence of the central wavelength 
was also characterised, showing a small positive shift of $65 \mathrm{pm} /{ }^{\circ} \mathrm{C}$ in the temperature range of $15-50{ }^{\circ} \mathrm{C}$, as shown in the inset of Fig. 2. Moreover, the filter $-3 \mathrm{~dB}$ bandwidth was constant within $<100 \mathrm{pm}$ for the measured temperatures, which corresponds to a variation of less than $1.6 \%$. The robustness, small size, and excellent stopband rejection of the device are remarkable advantages compared to other state-of-the-art filters [1-4].

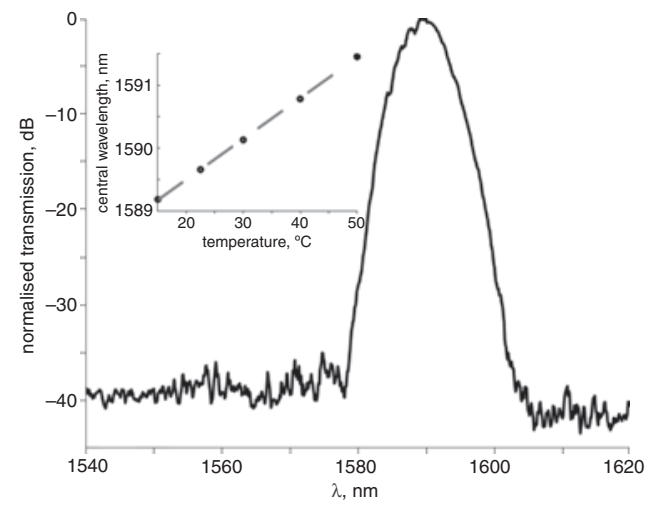

Fig. 2 Optical micrograph image of fabricated two-stage filter

Central wavelength $\lambda_{c}$ focused in position $\mathrm{F}$ of Rowland circle and guided to the input of the second filtering stage

Additional filter designs with central wavelengths shifted down to $1520 \mathrm{~nm}$ were measured. A slight deterioration in the performance of the device was observed for shorter central wavelengths, owing to an increase in the losses of the coupling system and the device itself, as well as to limits in the operational range of the subwavelength AR structure. Nonetheless, for each $10 \mathrm{~nm}$ shift in central wavelength in the $1520-1590 \mathrm{~nm}$ range, the measured intrinsic loss and extinction ratio penalties were below $0.1 \mathrm{~dB}$ and $0.55 \mathrm{~dB}$, respectively.

Conclusion: A two-stage bandpass filter with blazed waveguide sidewall gratings has been designed and fabricated, showing a $-3 \mathrm{~dB}$ bandwidth of $6.2 \mathrm{~nm}$ at a central wavelength of $1590 \mathrm{~nm}$ and a stopband rejection of $40 \mathrm{~dB}$, as well as a good temperature stability.
Acknowledgment: Financial support from the Spanish Ministry of Science and Innovation is acknowledged under grant TEC2008-04105

(C) The Institution of Engineering and Technology 2012

5 March 2012

doi: $10.1049 / \mathrm{el} .2012 .0591$

One or more of the Figures in this Letter are available in colour online.

A.V. Velasco and M.L. Calvo (Dpto. de Optica, Facultad de Ciencias Fisicas, Universidad Complutense de Madrid, Madrid 28040, Spain)

E-mail: avillafr@pdi.ucm.es

P.J. Bock, P. Cheben, J.H. Schmid, J. Lapointe, D.-X. Xu, S. Janz and A. Delâge (Institute for Microstructural Sciences, National Research Council Canada, Ottawa, Ontario K1A 0R6, Canada)

\section{References}

1 Zhu, L., Huang, Y., and Yariv, A.: 'Integration of a multimode interference coupler with a corrugated sidewall Bragg grating in planar polymer waveguides', IEEE Photonics Technol. Lett., 2006, 18, (6), pp. $740-742$

2 Madsen, C.K.: 'Efficient architectures for exactly realizing optical filters with optimum bandpass designs', IEEE Photonics Technol. Lett., 1998, 10, (8), pp. $1136-1138$

3 Cheben, P.: 'Wavelength dispersive planar waveguide devices' in 'Optical waveguides: from theory to applied technologies' (CRC Press, 2007), pp. 173-230

4 Mizuno, T., Oguma, M., Kitoh, T., Inoue, Y., and Takahashi, H.: 'Lattice-form CWDM interleave filter using silica-based planar lightwave circuit', IEEE Photonics Technol. Lett., 2006, 18, (15), pp. $1570-1572$

5 Hao, Y., Wu, Y., Yang, J., Jiang, X., and Wang, M.: 'Novel dispersive and focusing device configuration based on curved waveguide grating', Opt. Express, 2006, 14, (19), pp. 8630-8637

6 Bock, P.J., Cheben, P., Delâge, A., Schmid, J.H., Xu, D.-X., Janz, S., and Hall, T.J.: 'Demultiplexer with blazed waveguide sidewall grating and sub-wavelength grating structure', Opt. Express, 2008, 16, (22), pp. $17616-17625$

7 Schmid, J.H., Cheben, P., Janz, S., Lapointe, J., Post, E., and Xu, D.-X.: 'Gradient-index antireflective subwavelength structures for planar waveguide facets', Opt. Lett., 2007, 32, (13), pp. 1794-1796 